\title{
Gestão do conhecimento e sistemas de informação: uma análise sob a ótica da teoria de criação do conhecimento
}

Cláudia Andressa Cruz

\begin{abstract}
Mestre em Engenharia de ProduçãoEscola de Engenharia de São Carlos Universidade de São Paulo
\end{abstract}

Marcelo Seido Nagano

Professor Doutor do Departamento de Engenharia de Produção, Escola de Engenharia de São Carlos - Universidade de São Paulo

O objetivo deste artigo é analisar como os sistemas de informação podem contribuir para uma gestão do conhecimento eficiente. Para tanto, foi desenvolvida uma pesquisa exploratória em empresas residentes a parques tecnológicos do estado de São Paulo, a qual evidenciou o suporte que sistemas de informação prestam à gestão do conhecimento. Com isso foi possível delinear mecanismos para suprir a deficiência de alguns sistemas.

Palavras-chave: Gestão do conhecimento; Sistemas de informação; Parques tecnológicos.

\section{Knowledge Management and Information systems: an analyzes about knowledge creation theory}

The concept of information retrieval is in the origins of Information Science, being responsible for a remarkable technological approach. When the concern about the systems' users is introduced IS is influenced by cognitive sciences, which do not favor social interaction. The article reflects about cognitivistic presuppositions about analyses of Information Retrieval Systems and presents a perspective from the authors who consider the need of a social approach as important as technological development. 


\section{Keywords: Knowledge management; Information systems; Science park.}

Recebido em 27.12.2007 Aceito em 18.06.2008

\section{Introdução}

A evolução do pensamento administrativo é consagrada por diversos estudos gerenciais, que abordam as idéias predominantes em determinados momentos históricos e que prevalecem até hoje nas organizações, as quais vão desde a racionalização do trabalho, a visão humanística, a visão estruturalista-sistêmica, a perspectiva contingencial, até as teorias contemporâneas. Nestas últimas, é observada uma transição, na qual o poder de gerar toda a riqueza e o poder da sociedade atual deixa de provir, exclusivamente, dos fatores tradicionais de produção, tais como o capital, a terra e o trabalho; advindo também de bens intangíveis como o conhecimento organizacional (LEONARD-BARTON, 1998; MORGAN, 1996; SANTIAGO Jr., 2002; WREN, 1994).

Neste contexto, muitas empresas se deparam com o desafio de competir num mundo em que conhecimento, e não apenas os recursos naturais e a mão-de-obra abundante e barata, constitue vantagem competitiva (EDWARDS et al., 2003). Nonaka (1991) corrobora quando menciona que a única fonte de vantagem competitiva duradoura é o conhecimento. Desse modo, buscando vantagens competitivas, surgem teorias e abordagens visando gerenciar o conhecimento estrategicamente.

De acordo com uit Beijerse (1999), a gestão do conhecimento (G.C.) visa criar, disseminar, armazenar e incorporar às rotinas de trabalho todo o conhecimento desenvolvido na organização, utilizando a capacidade dos trabalhadores para interpretação de dados e informações (por recursos disponíveis). Nonaka (1991), por outro lado, analisa o gerenciamento do conhecimento como um ciclo de criação contínuo do conhecimento, em que se destacam as companhias que criam novos conhecimentos, os disseminam por toda a organização e rapidamente os incorporam em novos produtos, serviços e tecnologias.

A fim de que uma gestão efetiva seja alcançada, elementos de apoio à gestão do conhecimento são imprescindíveis. É neste âmbito que o presente trabalho aborda os sistemas de informação, por serem provedores de suporte técnico e funcionalidade que oferecem ao processo de gestão do conhecimento, no que concerne principalmente as funções de armazenamento e disseminação do conhecimento.

Com o intuito de perceber como os sistemas de informação viabilizam a gestão do conhecimento, foi realizada uma pesquisa de campo, junto às empresas instaladas em dois parques tecnológicos pertencentes ao Estado de São Paulo: o Parque Científico e Tecnológico de Campinas - Ciatec (Cia. de Desenvolvimento do Pólo de Alta Tecnologia de Campinas), localizado na cidade de Campinas; e o Parque Tecnológico de São Carlos - ParqTec, localizado na cidade de São Carlos. 
A escolha de uma amostra de pesquisa constituída por empresas residentes em Parques Tecnológicos remete à magnitude de conhecimento incorporado nestes. Tratam-se de empresas que estão constantemente inovando, formadas em sua maioria por pessoas altamente especializadas em sua área de atuação; ademais há transferência continua de conhecimento universidades-empresas devido ao contexto em que estão inseridas.

Dessa forma, este trabalho parte da hipótese de que uma infraestrutura adequada em sistemas de informação, com sistemas integrados, promove uma melhora na gestão do conhecimento, permitindo interações entre os membros organizacionais e acesso a inúmeras bases de dados, configurando-se na disseminação e armazenamento de informações e conhecimento. Almeja-se, com esta hipótese, identificar se sistemas de informação apropriados implicam necessariamente o aumento da criação, compartilhamento, armazenamento e incorporação do conhecimento organizacional.

\section{Gestão do Conhecimento}

Desde o período clássico grego, a história da filosofia pode ser vista como o processo de busca da definição da palavra conhecimento. 0 conceito conhecimento não é algo consensual. Conforme ressalta Nonaka (1994), o conhecimento pode ser definido como "uma crença justificadamente verdadeira". Ou ainda como denota Liebeskind (1996), conhecimento é uma informação cuja validade é estabelecida por meio de testes de veracidade.

Platão (apud MÜLLER-MERBACH, 2004) faz uso de uma analogia com o discurso direto para definir o conhecimento. O discurso direto é entendido como um diálogo dinâmico em que os participantes interpelam uns aos outros, reagem aos argumentos dos parceiros, respondem às questões, tentam convencer os demais, tudo baseado na percepção, convicção, compromissos, talvez até mesmo paixão. Assim, o conhecimento é entendido como parte da consciência humana.

$\mathrm{Na}$ literatura são apresentadas algumas tipologias que caracterizam o termo conhecimento, tais como: implícito, explícito, sistemático, informal, codificável e não codificável, entre outros. A mais comumente utilizada na literatura em geral é a proposta por Polanyi (apud Nonaka e Takeuchi, 1997), em que o autor identifica dois tipos de conhecimento: o conhecimento tácito e o conhecimento explícito.

O conhecimento tácito é altamente pessoal e difícil de formalizar, o que dificulta sua transmissão e compartilhamento. Palpites subjetivos, insights, intuições, valores e emoções fazem parte desta categoria (NONAKA, 1991). Por outro lado, o conhecimento explícito pode ser expresso em palavras e números, sendo facilmente comunicado e compartilhado sob a forma de dados brutos, fórmulas científicas, princípios universais, especificações e manuais. O conhecimento explícito 
pode ser facilmente transmitido, formalmente e sistematicamente, entre indivíduos (NONAKA e KONNO, 1998).

A criação do termo gestão do conhecimento teve seu início com a automação, por volta de 1970, em que os sistemas de computadores eram denominados "processadores de dados". Pretendia-se, com esta caracterização, conduzir a noção de partes de dados que alimentavam computadores, que os processava individualmente ou ainda em pequenos grupos. Após esta fase iniciou-se um processo em que os dados estavam combinados com uma estrutura e rótulos, 0 que configurou 0 "processamento de informações". A principal diferença desta fase com a anterior é, nesta, os dados não eram gerenciados de forma isolada, o que tornou possível estocá-los em tabelas, permitindo operações um pouco mais complexas.

A partir dos anos 90, um novo conjunto de aplicações emergiu, diferindo qualitativamente das fases antecessoras. Iniciou-se um processo de armazenamento de informações de toda parte da organização: financeira, demográfica, clientes, produtos, entre outros. Não surpreendentemente, este processo assumiu uma conotação que a distinguisse das demais, denominado "processamento de conhecimento". Neste âmbito, o aprimoramento da gestão da informação eleva-se à chamada gestão do conhecimento (DILLON, 2002).

Gestão do conhecimento envolve criar, captar, disseminar, armazenar, disponibilizar e incorporar os conhecimentos individuais, transformando-os em conhecimento organizacional disseminado dentro e fora da organização (NONAKA e TACKEUCHI, 1997).

O presente trabalho aprofunda seus estudos na teoria de criação do conhecimento organizacional desenvolvida por Nonaka (1991), notoriamente reconhecida pela literatura, por concentrar muitas das melhores práticas denotadas por outras perspectivas de G.C.

Nonaka e Toyama (2003) caracterizam a criação do conhecimento como um processo mutável e dialético de pensamento e ação, em que várias contradições são sintetizadas através de interações dinâmicas, passíveis de mudança, entre os indivíduos, a organização e o ambiente. Entenda-se síntese como uma integração de aspectos opostos através deste processo dinâmico entre o diálogo e a prática.

Segundo Oliveira Jr. (2001), partindo do pressuposto de que o sucesso de uma empresa está baseado em sua capacidade de criar e transferir conhecimento de forma mais eficaz que seus competidores, entender os mecanismos pelos quais o conhecimento pode ser criado e transferido na empresa é ponto de partida para um resultado superior.

De acordo com Nonaka e Takeuchi (1997); Nonaka, Umemoto e Senoo (1996), a organização deve ser capaz de captar, armazenar e transmitir o novo conhecimento organizacional criado a partir da transformação do conhecimento tácito (pessoal e informal) em conhecimento explícito (formal e sistemático).

Este processo de criação do conhecimento organizacional é denominado de SECI - Socialização, Externalização, Combinação e 
Internalização - e ocorre mediante a interação contínua e dinâmica entre o conhecimento tácito (informal e incorporado em valores e crenças) e o conhecimento explícito (formal e codificável) (NONAKA e KONNO, 1998).

O processo de criação de conhecimento denominado SECI, ilustrado na FIG. 1, é abordado por Nonaka e Toyama (2003), que afirmam ser o conhecimento criado mediante a contradição dos recursos internos e 0 ambiente.

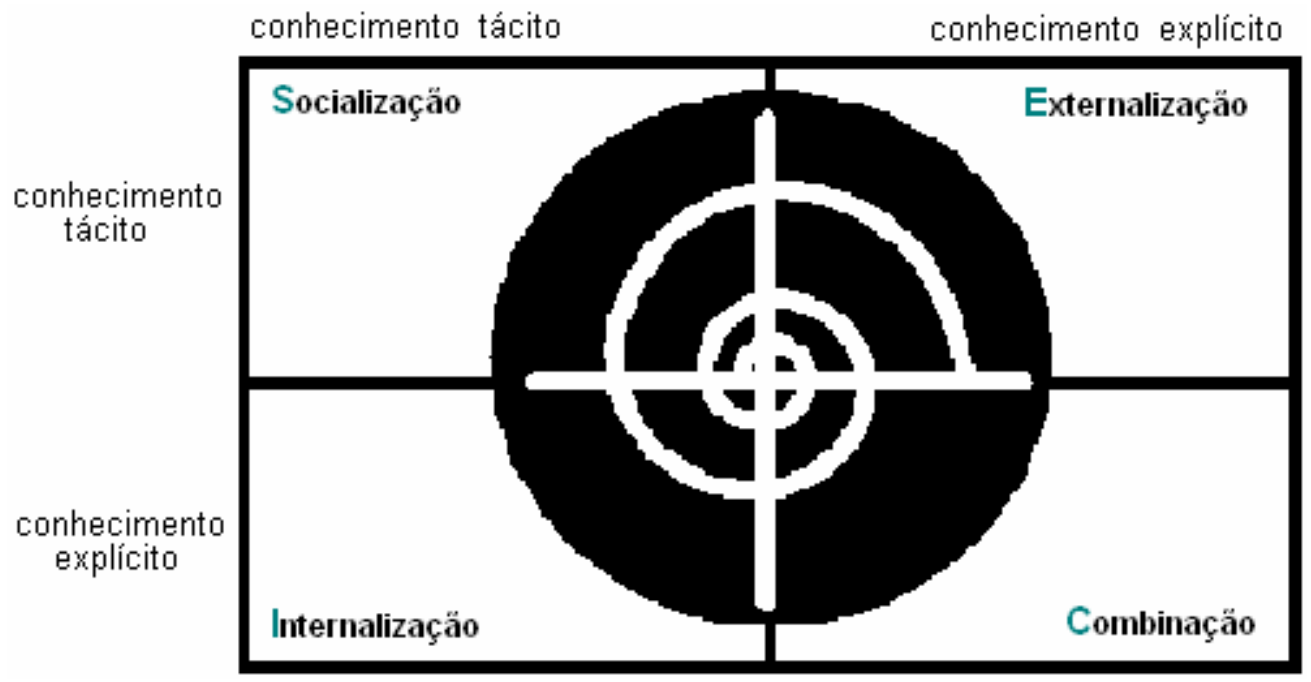

FIGURA 1: Processo SECI

Fonte: Adaptado de Nonaka e Takeuchi (1997:80)

De forma que a socialização é o processo de compartilhamento de experiências, criador do conhecimento tácito, que é articulado em conhecimento explícito na externalização, através do diálogo. A combinação é o processo de sistematização do conhecimento explícito em sistema de conhecimento, onde os indivíduos trocam e combinam conhecimento, que é incorporado na internalização através do "aprender fazendo", pela verbalização e diagramação. Quando a maioria dos membros da organização compartilha do novo modelo mental, o conhecimento é incorporado na cultura organizacional. $E$, a cada internalização efetiva, o ciclo reinicia, levando ao aperfeiçoamento ou à inovação.

\section{Sistemas de informação}

Os recursos da tecnologia de informação podem otimizar fases do processo SECI, seja por facilitar que as pessoas sejam encontradas, podendo ocorrer então a socialização e a externalização; por auxiliar no registro do conhecimento, na combinação; ou por agilizar o acesso ao conhecimento, na internalização (SILVA, 2002).

Os sistemas de informação geram, coletam, combinam, armazenam, codificam e disseminam dados, informações e conhecimento, caracterizando uma ferramenta que sistematiza o funcionamento dos 
processos que são estabelecidos por meio de uma estratégia predefinida (SCHULZ, 2001).

Devido ao suporte técnico e a toda funcionalidade que os sistemas de informação oferecem ao processo de gestão do conhecimento, ele pode ser utilizado como um recurso estratégico de apoio ao processo de gestão do conhecimento.

Assim, investimentos em tecnologias de informação e integração dos sistemas tornam-se indispensáveis, pela facilitação de transferência de informações, e, principalmente, conhecimento, sem contar o modo como facilitam o processo de codificação do conhecimento, configurando um importante aspecto estratégico (COWAN, DAVID e FORAY, 2000; COHENDET e STEINMUELLER, 2000; DAVENPORT, DE LONG e BEERS, 1998; HANSEN, NOHRIA, TIERNEY, 1999; HITT, IRELAND $\mathrm{C}$ HOSKINSSON, 2005; NONAKA, 1994; PRAHALAD e HAMEL, 1990; TERRA, 2001; VASCONCELOS, 2001; ZACK, 1999).

\section{Método de pesquisa}

A relação entre os sistemas de informação e a gestão do conhecimento, em empresas residentes em parques tecnológicos, é um tema pouco explorado na literatura, caracterizando uma pesquisa exploratória e descritiva; seja por realizar descrições categóricas da situação, visando descobrir as relações existentes entre os elementos componentes da mesma, seja por explorar um assunto pouco abordado, medindo as características descritas nas questões de pesquisa (CERVO e BERVIAN, 1983; DANE, 1990; GIL, 2002; HAIR JR. et al., 2005a; LAKATOS e MARCONI, 1992).

Esta pesquisa foi realizada junto às empresas residentes em dois dos parques tecnológicos do Estado de São Paulo: Cia. de Desenvolvimento do Pólo de Alta Tecnologia de Campinas - Ciatec; e Parque Tecnológico de São Carlos - ParqTec, o que possibilitou a comparação entre os dois grupos de empresas (Ciatec e ParqTec), no que se refere à gestão do conhecimento. Esses parques são essencialmente de base tecnológica, representando, em 2004, 94\% do total de parques brasileiros (em projeto, implantação e operação) (ASSOCIAÇÃO NACIONAL DE ENTIDADE PROMOTORAS DE EMPREENDIMENTO INOVADORES - ANPROTEC, 2004).

A população-alvo era composta por 39 empresas residentes nos dois parques tecnológicos, e sua composição por parque configura-se do seguinte modo:

I. 23 empresas no Cia. de Desenvolvimento do Pólo de Alta Tecnologia de Campinas (Ciatec);

II. 16 empresas no Parque Tecnológico de São Carlos - ParqTec.

A pesquisa nas empresas foi realizada por meio de um questionário fechado, que buscou captar e compreender o processo de gestão do conhecimento, ou como os sistemas de informação as subsidiavam. 
Assim, empregou-se a escala Likert para mensurar estes elementos. A escala está contida no intervalo de 1 a 7 , em que 1 corresponde a discordo totalmente e 7 a concordo plenamente. Neste âmbito, o tratamento dos dados e a análise procederam da seguinte forma: na primeira fase, as respostas foram sistematizadas, permitindo uma análise descritiva, em que é denotado o alfa de Cronbach, que objetiva a validação do questionário; depois os resultados foram cruzados, desenvolvendo-se uma análise de correspondência e o teste binomial.

\section{Análise dos Resultados}

Foram realizados três tipos de análise: primeiramente uma análise descritiva, visando denotar o comportamento das questões individualmente e a validação do questionário através do alpha de Cronbach; posteriormente, foi realizada a análise de correspondência, com o objetivo de demonstrar graficamente o comportamento dos atributos investigados perante os dois grandes grupos de empresas pesquisadas do ParqTec e do Ciatec; por fim, um teste binomial, visando validar ou refutar a hipótese $\mathrm{HO}$ de que as proporções de "discordância", "concordância" e "parcial" são iguais para os Parques de São Carlos e Campinas, ParqTec e Ciatec, respectivamente.

\section{Análise descritiva}

Após os dados contidos nos questionários serem coletados, foi verificada a integridade e a coerência das respostas, uma vez que incoerências, como respostas faltantes ou mais de uma alternativa assinalada, podiam ainda ser corrigidas. Tendo feito esta verificação, o passo seguinte foi tabular somente as respostas em uma planilha do Statistical Package for the Social Sciences (SPSS).

Dentre as 39 empresas pesquisadas, foram invalidados os questionários em quatro empresas do Ciatec e cinco do ParqTec, pois estas eram compostas por apenas 1 pessoa, impossibilitando as inferências sobre gestão do conhecimento. Em outras cinco empresas do Ciatec não foi possível estabelecer contato, uma vez que os e-mails retornaram e o telefone não foi atendido; e, por fim, quatro empresas do Ciatec optaram por não participar da pesquisa.

Totalizou assim uma amostra restante de 21 empresas, onze instaladas no ParqTec e dez empresas do Ciatec. A taxa de questionários aceitos sobre a amostra inicial de 16 empresas do ParqTec corresponde a $68,75 \%$ e sobre a amostra de 23 empresas do Ciatec é de 43,48\%.

Perante as respostas coletadas, obteve-se primeiramente o coeficiente alpha de Cronbach, uma etapa fundamental na primeira fase de análise dos dados, pois se refere à consistência das respostas dos indivíduos em relação a todos os itens investigados, além de não requerer separação de itens nem repetição da aplicação (BLAND e ATLMAN, 1997; SCHMITT, 1996). Foi obtido um alpha equivalente a 0,8426, que, de acordo com Hair et al. (2005b), indica uma confiabilidade muito boa, o 
que valida o questionário elaborado e aplicado no contexto desta pesquisa.

Com isso, construiu-se o histograma para cada variável (questão), obtendo-se o desvio padrão, a média e a freqüência das respostas. Neste âmbito foi possível observar uma boa distribuição na freqüência das respostas, não denotando tendências acentuadas.

\section{Análise de correspondência}

A correspondência apresenta-se como uma técnica de interdependência desenvolvida para facilitar tanto a redução dimensional (SECI e Sistemas de Informação) da classificação de objetos (os Parques tecnológicos: Ciatec e Parqtec) em um conjunto de atributos (socialização, externalização, combinação, internalização e sistemas de informação), quanto o mapeamento perceptual de objetos relativo a esses atributos (HAIR et al., 2005a).

A técnica de correspondência foi aplicada por meio do software The SAS System, que exibe de forma gráfica as respostas dadas pelos grupos das empresas entrevistadas, ParqTec e Ciatec.

Os gráficos demonstram a proximidade ou o afastamento dos grupos (S - ParqTec e C - Ciatec) sobre determinado atributo, o qual é composto por conjuntos de questões. Por exemplo, R24: R indica resposta, 2 se refere à questão número dois e 4 corresponde à escolha do número quatro na escala Likert utilizada no questionário.

O GRAF. 1 apresenta a dispersão das respostas no que concerne ao processo SECI nos parques tecnológicos. 


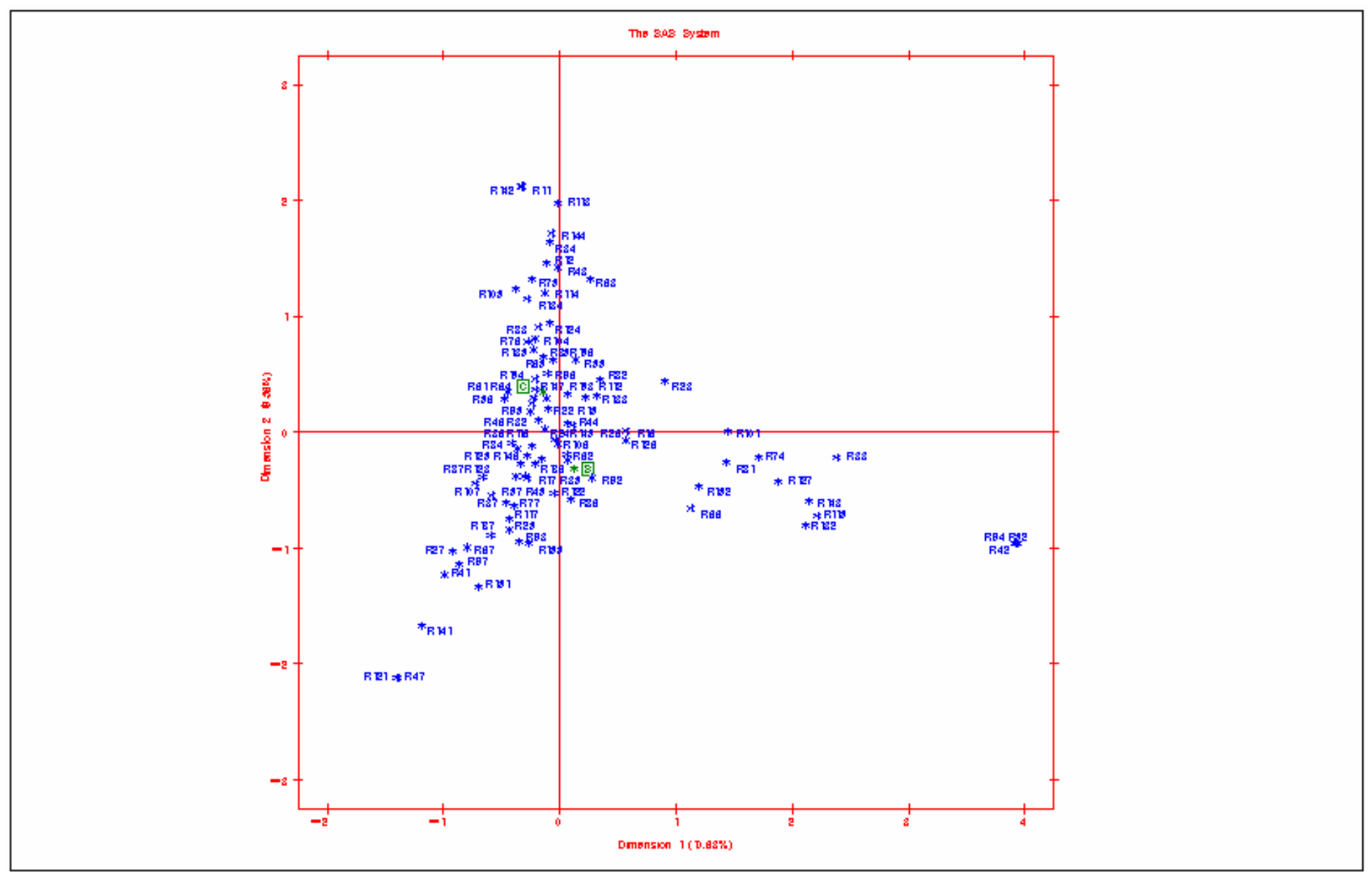

GRÁFICO 1: Análise de correspondência para o SECI

Fonte: Adaptado de CRUZ (2007) 
A análise de correspondência do SECI permite observar que os parques, assinalados no GRÁF. 1 pelas letras $(C)$ e $(S)$, referindo-se ao Ciatec e ParqTec, respectivamente, estão afastados, mostrando que, em relação ao processo de gestão do conhecimento SECI, as práticas empregadas pelas empresas instaladas nos parques diferem.

Os sistemas de informação, por sua vez, foi a dimensão que apresentou maior proximidade entre os grupos investigados, conforme GRÁF. 2, denotando que, de modo geral, os parques compartilham a mesma opinião. Foram explorados, nesta dimensão, os investimentos destinados às tecnologias de informação e à integração dos sistemas de informação, o que expressou um comportamento similar entre Ciatec e ParqTec, devido, provavelmente, à atuação tecnológica de ambas.

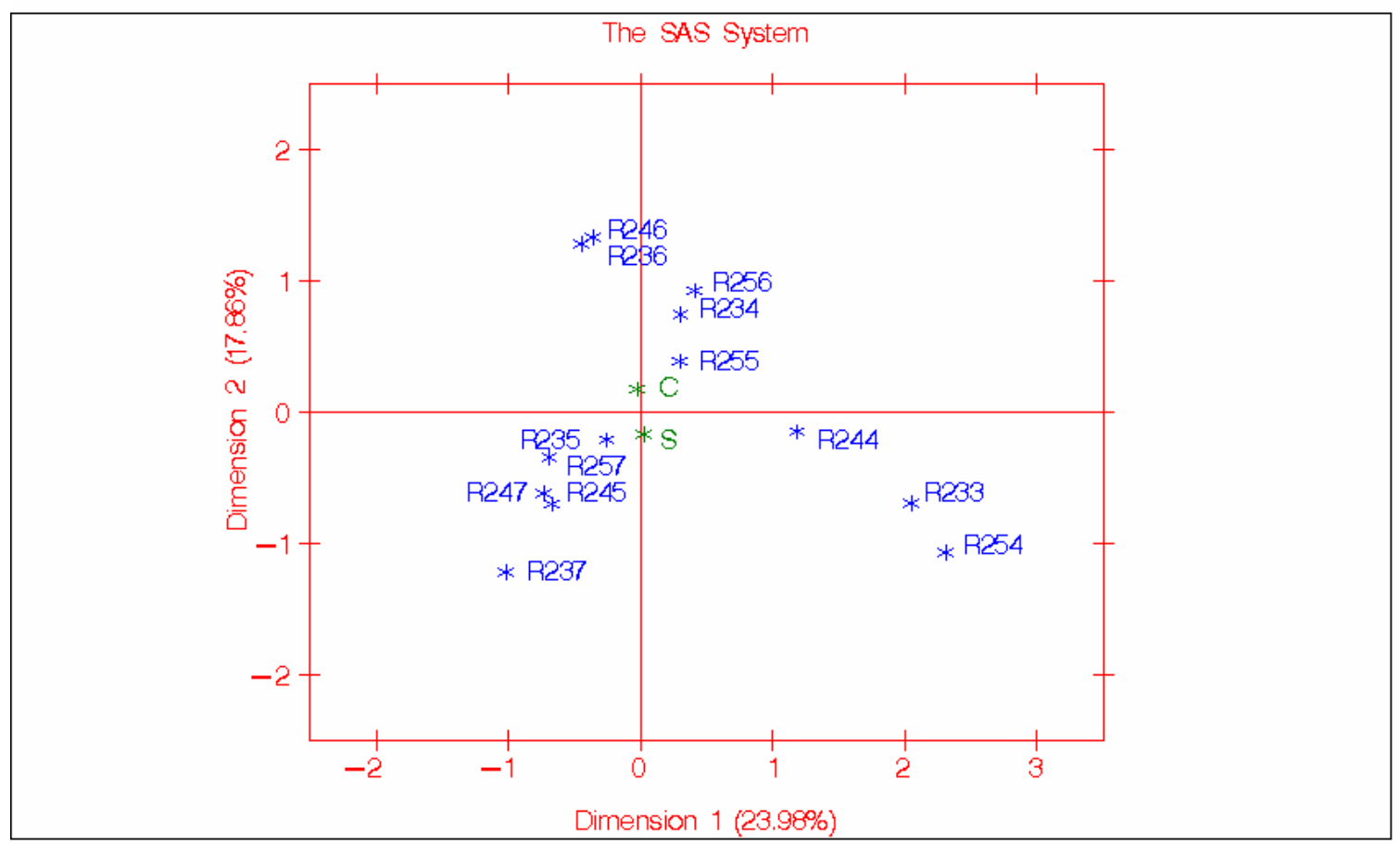

GRÁFICO 2: Análise de correspondência para os Sistemas de Informação Fonte: Adaptado de CRUZ (2007)

É provável que esta maior proximidade entre os grupos, no que se refere aos sistemas de informação, advenha da semelhança entre os parques; uma vez que são compostos por empresas de base tecnológica, que requerem uma infra-estrutura parecida e estão inseridas em ambientes similares de mão-de-obra especializada, formada em sua maioria por técnicos e engenheiros que possuem um conhecimento tácito acumulado importante.

\section{Teste binomial}

O teste binomial é usado para fazer inferências de diferenças entre duas populações, baseadas em dados de duas amostras aleatórias. Assim, os dados experimentais utilizados pelo teste são as freqüências relativas p 
e $\mathrm{q}$, referentes às duas alternativas possíveis naquele determinado experimento. A freqüência esperada para $p$ e $q$, em caso de igualdade perfeita, seria $1 / 2$ para ambos. Como, num experimento, dificilmente $p$ é igual a q, o teste avalia, em última análise, até que ponto os valores de $p$ e q podem diferir, sem deixarem de ser estatisticamente iguais. Dessa forma, o teste fornece o $\mathrm{p}$-value, que informa se a proporção amostral de diferenças é significativa, isto é, se HO é a hipótese verdadeira (CAMPOS, 2001).

Neste contexto, o teste binomial, através do software Minitab 2000, foi utilizado como método de análise de proporção que compara as visões e opiniões entre os parques diferentes a respeito das mesmas questões.

Hipótese Científica:

HO: Proporções de "discordância", "parcial" e "concordância" são iguais para os parques de São Carlos e Campinas.

H1: Proporções de "discordância", "parcial" e "concordância" são diferentes para os parques de São Carlos e Campinas.

Hipótese Estatística:

$$
H_{0}:\left(\begin{array}{l}
P 1_{p 1} \\
P 2_{p 1} \\
P 3_{p 1}
\end{array}\right)=\left(\begin{array}{l}
P 1_{p 2} \\
P 2_{p 2} \\
P 3_{p 2}
\end{array}\right) \quad H_{1}:\left(\begin{array}{l}
P 1_{p 1} \\
P 2_{p 1} \\
P 3_{p 1}
\end{array}\right) \neq\left(\begin{array}{l}
P 1_{p 2} \\
P 2_{p 2} \\
P 3_{p 2}
\end{array}\right)
$$

Tal que P1 é a proporção de empresas do parque que discorda, P2 é a proporção de empresas do parque com opinião parcial e P3 é a proporção de empresas do parque que concorda.

A TAB. 1 mostra o comportamento de cada questão perante a comparação entre os dois Parques: ParqTec (p1) e Ciatec (p2).

TABELA 1 - Comparação: Parque de São Carlos e Campinas

\begin{tabular}{ccccc}
\hline Questões & p-value P1 & p-value P2 & p-value P3 & Resultado \\
\hline 1 & 0,943 & $*$ & 0,943 & Aceito \\
2 & 0,279 & 0,015 & 0,269 & Rejeito \\
3 & 0,476 & 0,593 & 0,890 & Aceito \\
4 & 0,696 & 0,696 & 0,505 & Aceito \\
5 & 0,329 & $*$ & 0,329 & Aceito \\
6 & 0,407 & 0,476 & 0,801 & Aceito \\
7 & $*$ & 0,476 & 0,476 & Aceito \\
8 & 0,217 & 0,050 & 0,835 & Rejeito \\
9 & 0,034 & 0,329 & 0,015 & Rejeito \\
10 & 0,593 & 0,476 & 0,890 & Aceito \\
11 & 0,020 & 0,314 & 0,284 & Rejeito \\
12 & 0,217 & 0,269 & 0,864 & Aceito \\
13 & 0,072 & 0,476 & 0,256 & Aceito \\
14 & 0,157 & 0,119 & 0,757 & Aceito \\
15 & 0,104 & 0,537 & 0,269 & Aceito \\
16 & 0,593 & 0,916 & 0,757 & Aceito \\
17 & $*$ & 0,757 & 0,757 & Aceito \\
18 & $*$ & 0,943 & 0,943 & Aceito \\
\hline
\end{tabular}

Fonte: Adaptado de CRUZ (2007). 
Nota: $p$-value $\leq 0,05$. O asterisco $(*)$ representa que as respostas das empresas foram exatamente iguais.

Observa-se que apenas quatro questões rejeitaram HO, mostrando que as proporções de "discordância", "concordância" e "parcial" são diferentes para os parques de São Carlos e Campinas, no que concerne às estas quatro questões.

As questões rejeitadas foram: 2, 8, 9 e 11, as quais correspondem às variáveis: treinamento, interações coletivas e virtuais, banco de dados e elaboração de relatórios, respectivamente. Pode-se observar que uma delas está inserida na fase de socialização e as demais na combinação.

As empresas residentes no Parque Científico Tecnológico de Campinas - Ciatec demonstraram que a prática de treinamento, por meio de cursos extras, é pouco empregada. Provavelmente isso decorre do fato de serem empresas que tiveram sua fundação recente em relação àquelas do ParqTec e, por isso, querem que seus funcionários já tenham uma qualificação alinhada à sua função, sem a necessidade de investimentos extras da empresa no seu treinamento. Ademais, o Ciatec é uma fundação recente, denotando uma estrutura que está em crescimento quanto à organização de treinamentos e cursos para as empresas residentes.

As interações coletivas e virtuais, por sua vez, são mais empregadas pelo Ciatec, fato que pode ser explicado pelo maior número de funcionários das empresas, pois, no Ciatec, das dez empresas pesquisadas $50 \%$ possuem mais de cinco funcionários, o que torna as interações coletivas e virtuais indispensáveis para a execução dos projetos. Por outro lado, dentre as onze empresas entrevistadas do ParqTec, apenas $20 \%$ possuem mais de cinco funcionários, acarretando interações muito mais individualizadas.

Embora mais de $50 \%$ das empresas instaladas no ParqTec concordem com o acesso ao banco de dados da empresa, as empresas investigadas do Ciatec apresentaram $100 \%$ de utilização dos bancos de dados, o que acarretou a rejeição da hipótese HO. O tamanho das empresas, segundo o critério por número de funcionários, pode explicar este fato, mas é provável que a cultura de incentivo ao acesso aos bancos de dados seja muito mais intensa nas empresas do Ciatec.

Por outro lado, a elaboração de relatórios é uma prática mais ativa no ParqTec, tornando assim o conhecimento sistematizado disponível para necessidades futuras, evitando a perda de tempo em tarefas já executadas em projetos passados.

O teste binomial permitiu indicar as principais divergências entre as empresas pesquisadas no ParqTec e do Ciatec. Observou-se que, assim como na análise de correspondência, as diferenças são razoáveis entre os dois grupos investigados. Como foi verificado pela indicação do asterisco na TAB. 1 , houve seis casos, dentre os 75 , correspondendo a $8 \%$ de respostas, exatamente iguais entre os Parques, e apenas $16 \%$ das questões demonstraram diferenças entre os grupos. Ademais, as variáveis 
que compõem a fase combinação foram aquelas que geraram o maior distanciamento entre o ParqTec e o Ciatec.

\section{Considerações Finais}

Conforme Teece (2000) destaca, três objetivos são fundamentais para caracterizar o movimento da gestão do conhecimento:

- a criação de "depósitos" de conhecimento com informação externa (inteligência competitiva e melhores práticas) e com informação interna (relatórios de pesquisas internas); e conhecimento interno informal como discussões em bases de dados.

- o aprimoramento do acesso ao conhecimento, e com isso a reutilização deste por meio do desenvolvimento de ferramentas analíticas com interfaces amigáveis.

- valorização do ambiente de conhecimento organizacional, incluindo a espontaneidade dos indivíduos para compartilhar o seu conhecimento e experiências.

O intuito foi de compreender a gestão do conhecimento e o suporte que os sistemas de informação fornecem a esta gestão no ambiente de parques tecnológicos. O processo de gestão do conhecimento, por exemplo, foi subdividido em quatro variáveis macros: Socialização, Externalização, Combinação e Internalização, (SECI). Elas são caracterizadas pelos atributos: (S) interação face-a-face e individual, treinamento, confiança e comprometimento; (E) interações coletivas e face-a-face, conceitos de produtos/analogia, incentivo à criatividade e diálogo; (C) interações coletivas e virtuais, banco de dados, redes de comunicação, elaboração de relatórios; (I) interações individuais e virtuais, cultura organizacional disseminada, know how técnico e learning by doing. Enquanto que os sistemas de informação foram subdivididos em: sistemas de informação integrados, investimentos em T.I., redes de comunicação.

Dentre as vinte e uma empresas estudadas, das quais dez estão instaladas no Ciatec e onze no ParqTec, constatou-se, conforme a análise de correspondência, que, de maneira geral, não houve grandes disparidades na avaliação dos elementos integrantes do processo SECI e dos sistemas de informação. O que permite afirmar, no que se refere à gestão estratégica do conhecimento, que os comportamentos dos parques são, de uma forma geral, similares.

Entretanto, o teste binomial demonstrou que a intensidade dos atributos treinamento, interações coletivas e virtuais, banco de dados e elaboração de relatórios, é divergente entre os parques. O atributo treinamento caracteriza a fase de socialização e foi observado que as empresas residentes no Ciatec demonstraram que a prática de treinamento, por meio de cursos extras, são pouco empregadas, provavelmente pelo fato de serem empresas que tiveram sua fundação 
recente em relação àquelas do ParqTec, e por isso anseiam que os seus funcionários já tenham uma qualificação alinhada à sua função, não necessitando de investimentos extras da empresa com o treinamento. Sem contar que a maioria das empresas é composta por pequenas empresas nascentes, que buscam, em princípio, a sobrevivência; portanto os recursos são escassos para investimentos em treinamento (GREINER, 1998; LEONE, 1999). Ademais, o Ciatec é uma fundação recente, denotando uma estrutura de parque que está em crescimento quanto à organização de treinamentos e cursos para as empresas residentes; ao contrário do ParqTec, que está mais consolidado.

As interações coletivas e virtuais, o acesso ao banco de dados e a elaboração de relatórios constituem a fase de combinação. Foi observado que as interações coletivas e virtuais são mais empregadas pelo Ciatec, o que pode ser explicado pelo número de funcionários das empresas, pois, no Ciatec, das dez empresas pesquisadas, 50\% possuem mais de cinco funcionários, o que torna as interações coletivas e virtuais indispensáveis para a execução dos projetos. Por outro lado, dentre as onze empresas entrevistadas do ParqTec, apenas duas possuem mais de 10 funcionários, acarretando interações muito mais individualizadas.

Embora mais de $50 \%$ das empresas instaladas no ParqTec concordem Quanto à importância do acesso ao banco de dados da empresa, as empresas investigadas do Ciatec apresentaram $100 \%$ de utilização dos bancos de dados. O tamanho das empresas, segundo o critério por número de funcionários, pode explicar este fato, mas é provável que a cultura de incentivo ao acesso aos bancos de dados seja muito mais intensa nas empresas do Ciatec.

Por outro lado, a elaboração de relatórios é uma prática mais ativa no ParqTec, tornando assim o conhecimento sistematizado disponível para necessidades futuras, evitando a perda de tempo em tarefas que já foram executadas em projetos passados. Atribui-se a isto o fato das empresas do Ciatec, em geral, serem nascentes, possuindo uma estrutura pouco formalizada (LEONE, 1999). Desse modo, quanto aos atributos mencionados, a hipótese $\mathrm{HO}$ foi rejeitada, evidenciando proporções diferentes de "discordância", "concordância" e "parcial" entre os parques Ciatec e ParqTec.

Fica claro que a gestão do conhecimento está, em geral, desestruturada nas empresas do ParqTec, por priorizarem apenas alguns fatores do SECI, como socialização e combinação, em detrimento de mecanismos que incentivem o compartilhamento do conhecimento por meio de interações coletivas e face-a-face, e que viabilizem a incorporação efetiva do conhecimento adquirido nas rotinas organizacionais. Contudo, os sistemas de informação devem fornecer subsídios que viabilizem uma gestão efetiva de conhecimento, e estes mecanismos são pouco empregados. O Ciatec, por sua vez, prioriza um pouco mais o processo SECI, mesmo que de modo informal; e os sistemas de informação são mais utilizados como ferramentas de suporte a gestão das empresas. 
Ambientes formados em sua maioria por pessoas com estilos muito técnicos, em geral não priorizam mecanismos intangíveis, como expressão de valores e reconhecimentos, muitas vezes por serem fatores que costumam ter efeito a longo prazo. Dessa forma, priorizam-se elementos de suporte à gestão, como investimentos nos sistemas de informação, que proporcionam resultados em um período menor, por exemplo.

Com base, nas diferenças entre os grupos de empresas, é possível denotar alguns argumentos que explicam este comportamento. Primeiramente, a heterogeneidade das empresas, uma vez que, além de estarem em ambientes distintos, mesmo possuindo recursos similares, apresentam, muitas vezes, diferenças consideráveis em: estilo de gestão, recursos financeiros, tipo de produto ou serviço, tipo de processo de desenvolvimento, entre outros aspectos, característicos das empresas. Além do que, a estratégia de muitas das empresas é a sobrevivência do negócio. Puderam ser observados casos de empresas recém-formadas, em que as despesas ainda eram maiores que as receitas; em que, portanto, a lucratividade ainda não era uma realidade, dificultando sobremaneira a inferência de práticas como a gestão estratégica do conhecimento, que exige um ambiente mais estruturado e consolidado. Surpreendentemente, apesar destas restrições, inserções informais de mecanismos de gestão estratégica do conhecimento, como a elaboração de relatórios e manuais que contribuem para o armazenamento do conhecimento nestas empresas, e investimento em sistemas integrados de comunicação, ocorrem no contexto destas empresas. são:

Alguns mecanismos que poderiam ser empregados pelas empresas

- conceder maior liberdade aos funcionários, principalmente no que concerne ao processo criativo; permitir o "tentar e falhar", uma vez que o aprendizado pode gerar um crescimento futuro importante. é claro que numa pequena empresa recém-formada o controle do dirigente ainda é muito forte, mas é interessante delegar as tarefas possibilitando, dentro de um leque de opções, a autonomia de criação para alguns funcionários.

- coletar e armazenar o maior número de informações e conhecimentos de projetos, produtos e serviços prestados anteriormente, a fim de que a replicação de algum projeto realizado em situação anterior não exija um novo dispêndio de tempo. Tornar o acesso a esta base de dados uma rotina.

- incentivar interações intra e inter equipes, coletivizando-as.

- incentivar a utilização do conhecimento nas rotinas organizacionais, permitindo que seja agregado valor a novos conhecimentos.

- disseminar uma cultura de comprometimento e harmonia com os funcionários, despertando sua confiança na empresa, com o intuito de que compartilhem conhecimento com os demais, sem temer a substituição. 
É importante destacar algumas limitações encontradas nesta pesquisa e sugestões para trabalhos futuros. Embora significativas perante a população-alvo, o tamanho da amostra ainda foi um fator limitador, acarretando assim em resultados de pesquisa que não podem ser generalizados. Outra limitação foi o contato restrito da pesquisadora com os dirigentes das empresas, o que não permitiu a exploração de determinados aspectos. As respostas advindas de questionário também são fatores limitantes, por dependerem da honestidade dos entrevistados para compartilhar a situação real vivenciada nas empresas.

A análise quantitativa, por sua vez, também se evidencia como um limitante, uma vez que as organizações e seus contextos não se apresentam como uma ciência exata. É claro que a quantificação auxilia nas conclusões, mas não pode ser utilizada como única avaliação.

Poderão ser realizados trabalhos que auxiliem a sistematizar os pontos principais da gestão do conhecimento e das estratégias organizacionais, permitindo o auxílio destas ao processo de G.C.. Esta recomendação surge a partir de uma das limitações deste trabalho, que sintetizou os principais elementos com base em uma bibliográfica ainda restrita.

Ademais, sugerem-se estudos que contemplem a realidade das empresas instaladas nos parques tecnológicos, permitindo que práticas e ferramentas organizacionais, em sua maioria derivadas das grandes empresas, sirvam de base para o aprimoramento e a adequação de técnicas compatíveis com a realidade de pequenas empresas.

\section{Referências}

ASSOCIAÇÃO NACIONAL DE ENTIDADE PROMOTORAS DE EMPREENDIMENTO INOVADORES - ANPROTEC. Panorama 2004. Brasília: ANPROTEC, 2004.

BLAND, J. M.; ALTMAN, D. G. Statistics notes. BMJ, v.3, n.14, p.571-572, 1997 Disponível em: <http://bmj.bmjjournals.com/cgi/reprint/314/7080/572>. Acesso em: 03 jan. 2006.

CAMPOS,G.M. Estatística prática para docentes e pós graduandos. 2001. Disponível em: $<$ http://www.forp.usp.br/restauradora/gmc/gmc livro/gmc livro cap19.h tml>. Acesso em: 05 nov. 2006.

CERVO, A. L.; BERVIAN, P. A. Metodologia científica: para uso dos estudantes universitários. 3. ed. São Paulo: McGraw-Hill do Brasil, 1983.

COHENDET, P.; STEINMUELLER, W. E. The codification of knowledge: a conceptual and empirical exploration. Industrial and Corporate Change, v.9, n.2, p.195-209, 2000. 
COWAN, R.; DAVID, P. A.; FORAY, D. The explicit economics of knowledge codification and tacitness. Industrial and Corporate Change, v.9, n.2, p.211-253, 2000.

CRUZ, C. A. Gestão estratégica do conhecimento: estudo exploratório em empresas instaladas nos parques tecnológicos do Estado de São Paulo. Dissertação (Mestrado em Engenharia) - Escola de Engenharia de São Carlos, Universidade de São Paulo, São Carlos, 2007.

DANE, F. C. Research methods. Belmont - CA: Brooks/Cole, 1990.

DAVENPORT, T. H.; DE LONG, D. W.; BEERS, M. C. Successful knowledge management projects. Sloan Management Review, v.39, p.43-57, 1998.

DILLON, M. Knowledge management: chimera or solution? Libraries and the Academy, v.12, n.2, p.321-336, 2002.

EDWARDS, J. S. et al. Knowledge management research \& practice: visions and directions. Knowledge Management Research \& Practice, n. 1, p. 49-60, 2003.

GIL, A. C. Como elaborar projetos de pesquisa. 4.ed. São Paulo: Atlas, 2002.

GREINER, L. E. Evolution and revolution as organizations grow. Harvard Business Review, p.55-67, 1998.

HAIR JR., J. F. et al. Análise multivariada de dados. Porto Alegre: Bookman. 2005a.

- Fundamentos de métodos de pesquisa em administração. Porto Alegre: Bookman. 2005b.

HANSEN, M. T.; NOHRIA, N.; TIERNEY, T. What's your strategy for managing knowledge? Harvard Business Review. v.77, p.106-116. Mar./Apr, 1999.

HITT, M. A.; IRELAND, R. D.; HOSKINSSON, R. E. Administração estratégica: competitividade e globalização. 4 ed. São Paulo: Pioneira Thomson Learning, 2005.

LAKATOS, E. M.; MARCONI, M. A. Metodologia do trabalho científico. 4.ed. São Paulo: Atlas, 1992.

LEONARD-BARTON, D. Wellspring of Knowledge. Boston, MA: Harvard Business School Press, 1998.

LEONE, N. M. C. P. G. As especificidades das pequenas e médias empresas. Revista de administração, São Paulo, v.34, n.2, p.91-94, 1999.

LIEBESKIND, J. P. Knowledge, strategy, and the theory of the firm. Strategic Management Journal, v. 17, winter special issue, p. 93-107, 1996.

MORGAN, G. Imagens da organização. São Paulo: Atlas, 1996. 
MÜLLER-MERBACH, $\mathrm{H}$. Knowledge is more than information. Knowledge Management Research \& Pratice, n. 2, p.61-62, 2004.

NONAKA, I. The knowledge-creating Company. Harvard Business Review, p. 2-9, Nov./ Dec 1991.

- A dynamic theory of organizational knowledge creation. Organization Science, v.5, n.1, p.14-37, 1994.

NONAKA, I.; KONNO, N. The concept of ba: building a foundation for knowledge creation . California Management Review, v.40, n.3, p.40-55. 1998.

NONAKA, I.; TAKEUCHI, H. Criação de Conhecimento na Empresa: como as empresas japonesas geram a dinâmica da inovação. 13 ed., Rio de Janeiro: Elsevier, 1997.

- The theory of the knowledge-creating firm: subjectivity, objectivity and synthesys. Industrial and Corporate Change, v.14, n.3, p.419-436, 2005.

NONAKA, I..; TOYAMA, R. The knowledge-creating theory revisited: knowledge creation as a synthesizing process. Knowledge Management Research \& Practice, p.2-10, 2003.

NONAKA, I.; TAKEUCHI, H.; KONNO, N. SECI, Ba and Leadership: a unified model of dynamic knowledge creation. Long Range Planning, v.33, p.5-34, 2000.

NONAKA, I.; TAKEUCHI,; NAGATA, A. A Firm as a Knowledge-creating Entity: a new perspective on the Theory of the firm. Industrial and Corporate Change, v.9, n.1, p.1-20, 2000.

NONAKA, I.; UMEMOTO, K.; SENOO, D. From information processing to knowledge creation: a paradigm shift in business management. Technology in Society, v. 18, n.2, p.203-218, 1996.

OLIVEIRA JR.,. M. M. Competências Essenciais e Conhecimento na Empresa. In: FLEURY, M. T. L.; OLIVEIRA Jr, M. M. (Org). Gestão Estratégica do Conhecimento. São Paulo: Atlas, 2001. p.121-156.

POLANYI, M. The Tacit Dimension. Londres: Routledge\&Kegan Paul. 1966. citado por NONAKA, I.; TAKEUCHI, H. Criação de Conhecimento na Empresa: como as empresas japonesas geram a dinâmica da inovação. 13 ed. Rio de Janeiro: Elsevier, 1997.

PRAHALAD, C. K. e HAMEL, G. The core competence of the Corporation. Harvard business review, mai-jun, p.79-91. 1990

SANTIAGO JR, J. R. S. O Desenvolvimento de uma metodologia para a gestão do conhecimento em uma empresa de construção civil. Dissertação (Mestrado) - Escola Politécnica, Universidade de São Paulo. São Paulo, 2002. 
SCHMITT, N. Uses and abuses of coefficient alpha. Psychological Assessment, v.8, n.4, p.350-353, 1996. Disponível em: $<$ http://www.psych.upenn.edu/courses/psych600 303 Spring2003/600le c10a.pdf>. Acesso em: 03 jan. 2006.

SCHULZ, M. The uncertain relevance of newness: organizational learning and knowledge flows. Academy of Management Journal, v.44, n.4, p.661$681,2001$.

SILVA, S. L. Proposição de um modelo para caracterização das conversões do conhecimento no processo de desenvolvimento de produtos. Tese (Doutorado em Engenharia) - Escola de Engenharia de São Carlos, Universidade de São Paulo, São Carlos, 2002.

TEECE, D. J. Strategies for managing knowledge assets: the role of firm structure and industrial context. Long Range Planning, v.33, p.35-54, 2000.

TERRA, J. C. C. Gestão do Conhecimento: o grande desafio empresarial: uma abordagem baseada no aprendizado e na criatividade. São Paulo: Negócio, 2001.

uit BEIJERSE, R. P. Questions in knowledge management: defining and conceptualising a phenomenon. Journal of Knowledge Management, v.3, n.2, p.94-109, 1999.

VASCONCELOS, F. C. Da gestão do conhecimento à gestão da ignorância: uma visão co-evolucionária. Revista de Administração de Empresas, v.41, n.4, p.98-102, 2001.

ZACK, M. H. Developing a knowledge Strategy. California Management Review, v.41, n.3, p.125-145, 1999.

WREN, D. A. The evolution of management thought. 4.ed. New York: John Wiley \& Sons, Inc. 1994. 